\title{
Detecting Ecological Patterns Along Environmental Gradients: Alpine Treeline Ecotones
}

\section{The Harvard community has made this article openly available. Please share how this access benefits you. Your story matters}

\begin{tabular}{|l|l|}
\hline Citation & $\begin{array}{l}\text { Buckley, Hannah L., Bradley S. Case, Ronny Vallejos, J. Julio } \\
\text { Camarero, Emilia Gutiérrez, Eryuan Liang, Yafeng Wang, and } \\
\text { Aaron M. Ellison. 2016. “Detecting Ecological Patterns Along } \\
\text { Environmental Gradients: Alpine Treeline Ecotones." CHANCE 29 (2) } \\
\text { (April 2): 10-15. doi:10.1080/09332480.2016.1181960. }\end{array}$ \\
\hline Published Version & doi:10.1080/09332480.2016.1181960 \\
\hline Citable link & http://nrs.harvard.edu/urn-3:HUL.InstRepos:27409098 \\
\hline Terms of Use & $\begin{array}{l}\text { This article was downloaded from Harvard University's DASH } \\
\text { repository, and is made available under the terms and conditions } \\
\text { applicable to Other Posted Material, as set forth at http:// } \\
\text { nrs.harvard.edu/urn-3:HUL.InstRepos:dash.current.terms-of- } \\
\text { use\#LAA }\end{array}$ \\
\hline
\end{tabular}




\section{Detecting ecological patterns along environmental gradients: alpine treeline ecotones}

Hannah L. Buckley, Bradley S. Case, Ronny Vallejos, J. Julio Camarero, Emilia Gutiérrez, Eryuan Liang, Yafeng Wang, Aaron M. Ellison

Everyone is familiar with that age-old adage: "a picture is worth a thousand words". Among ecologists, the word "picture” easily could be replaced with the word "pattern”, although the significance remains the same: the pattern we observe in a single snapshot more than sums up what could be expressed if we tried to describe all the original events that led to the pattern. One particular class of patterns, spatial patterns, are the backbone of much contemporary ecological research. We often see recognizable and measurable structure in spatial patterns that tell a story about organisms and their histories: how interactions among individuals of the same or other species and between organisms and their environments have played out over time. An ongoing and challenging goal in ecology is determining how to adequately describe, visualize, and model such spatial patterns of organisms, their environments, and their inter-relationships (or covariances) so that we can make inferences about the types of processes that generated those patterns.

One of the main difficulties in pursuing this goal is dealing with the reality that the processes structuring spatial patterns in nature are complex. For example, trees in an unmanaged forest rarely are distributed at random (Figure 1a). Such spatial point patterns of individual trees and their attributes (such as height or diameter) may often be either 'over-dispersed' (Figure 1b) or clustered (Figure 1c) to varying degrees and in different ways depending on the spatial scale at which we measure them. Over-dispersion, or regularity, in tree spacing can occur due to processes such as competition for soil nutrients or shading effects. Alternately, we often observe clustered patterns where juvenile trees are found displaced from mature trees because the former are shaded out by the latter. As a result, seedlings and saplings often grow together in clusters within light gaps created when one or more mature trees die. In the forest stand illustrated in Figure 1c, the sizes of trees in each cluster are very similar: they display positive spatial autocorrelation. 

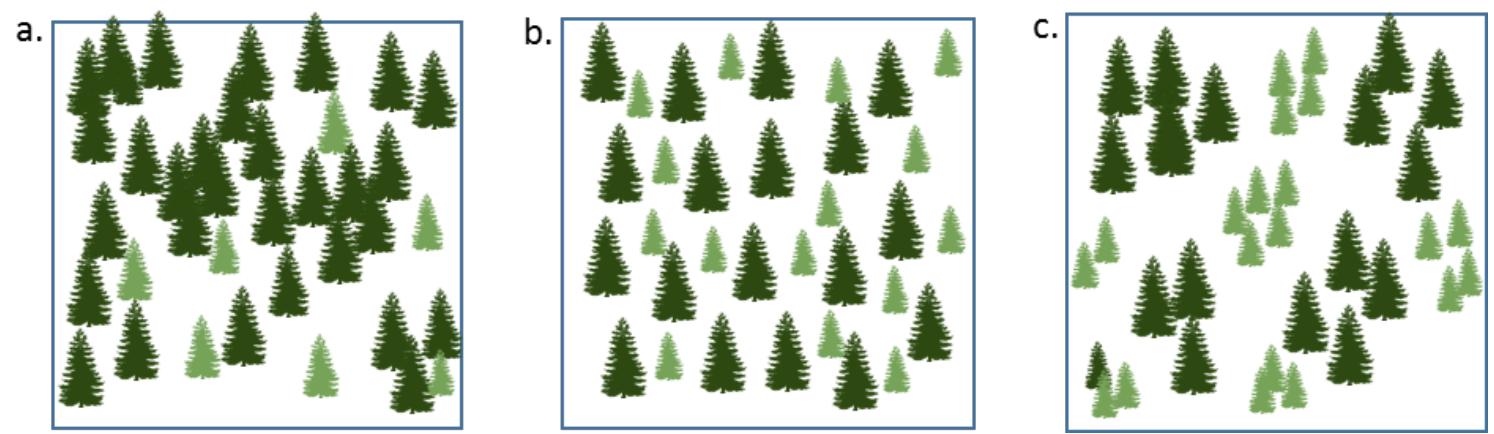

Figure 1. Forests with different structure in their spatial point patterns: (a) random; (b) overdispersed; (c) clustered.

One common way to describe spatial autocorrelation is with a variogram. A variogram illustrates the amount of variation in a measured attribute, such as size or age, as a function of the distance (or "lag") between all possible pairs of individuals in an area. The end result is a graph from which we can read the distance at which the spatial autocorrelation levels off (Figure 2). The variogram in Figure 2 illustrates that the spatial autocorrelation in tree density levels off at a distance of about 160 meters; thus, individuals are clustered at distances less than $160 \mathrm{~m}$ from each other, but become spatially independent from one another at larger lags.

a.

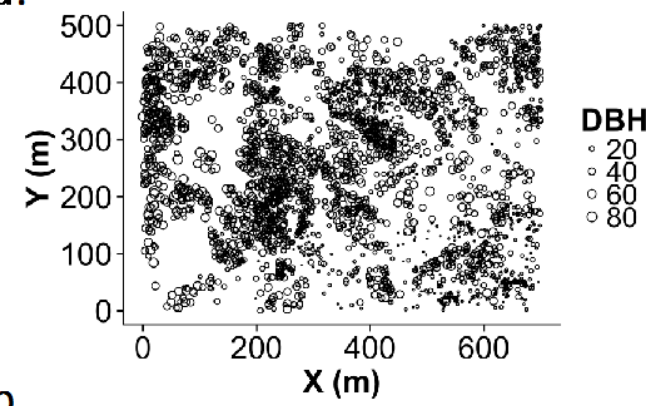

b.

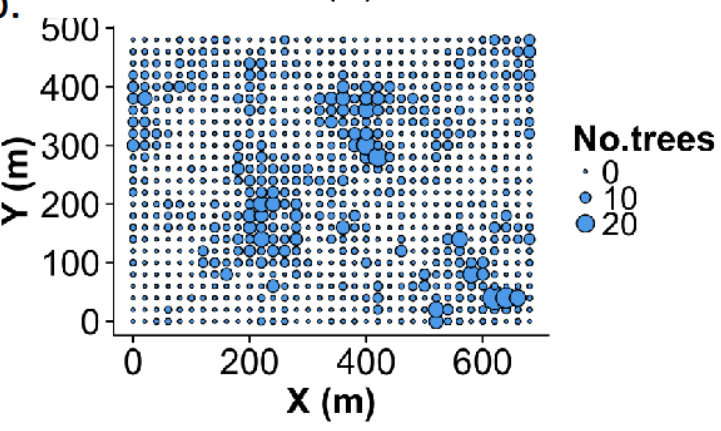

c.

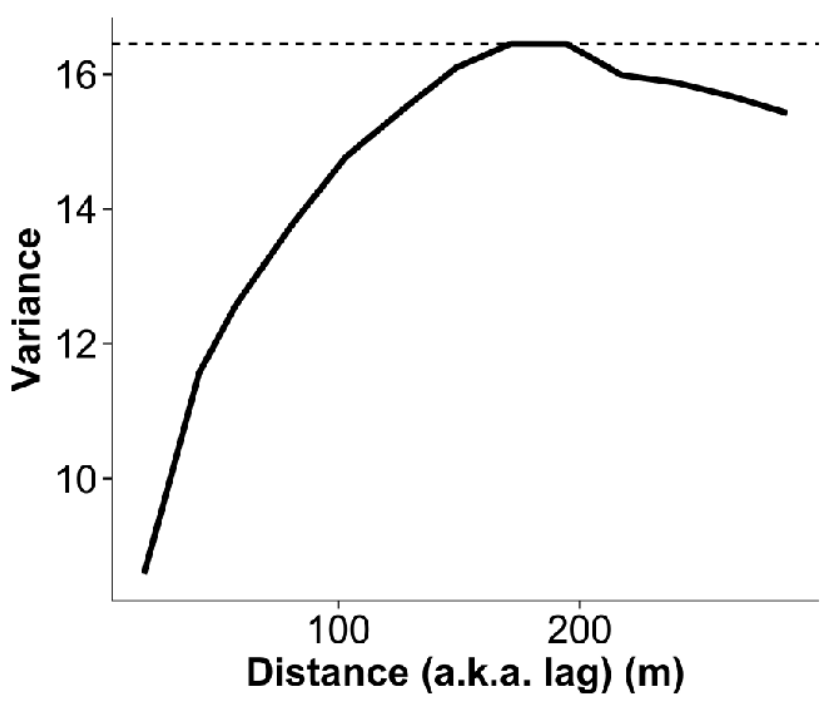


Figure 2. The variation in the spatial pattern of white pine trees within a large forest plot $(500 \times$ $700 \mathrm{~m}$ ) can be visually represented as (a) a spatial point pattern showing tree locations and their diameter at breast height (DBH) or (b) the number of trees (No. trees) within $20 \times 20$-m subplots. The variogram (c) shows the change in variance with distance of the number of trees within the sub-plots. The variogram shows that variance in the abundance of white pines between pairs of sub-plots increases with increasing inter-plot distances (also known as lags) up until $\approx 160 \mathrm{~m}$, where it begins to level off (horizontal dotted line). We conclude from the variogram that once sub-plots are separated by more than $160 \mathrm{~m}$ that there is no meaningful increase in the correlation in the abundance of white pines between the sub-plots.

A central assumption of a variogram is that the spatial pattern is isotropic: the estimated variance depends only on the distance between two measured objects, not the direction from one to another. However, in many situations, the processes that generate spatial patterns change in strength or character across an area. Such directional, or anisotropic, processes complicate the analysis of spatial patterns using variograms and other common methods (Bivand et al. 2008). A familiar example is a treeline: the transition from trees to alpine plants that we observe when walking up a mountain (Figure 3a). Below the treeline, trees are over-dispersed because they compete with one another for light and nutrients in the soil. But toward the treeline, the environment becomes much more stressful so that stunted trees, shrubs, and smaller plants tend to be clustered because there are fewer places that plants can grow successfully (Figure 3b).

a.

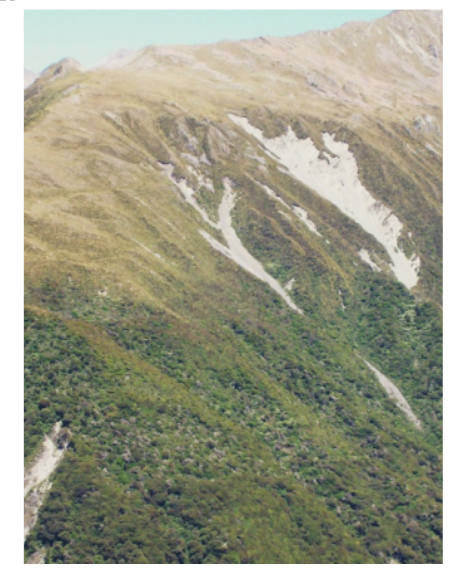

b.

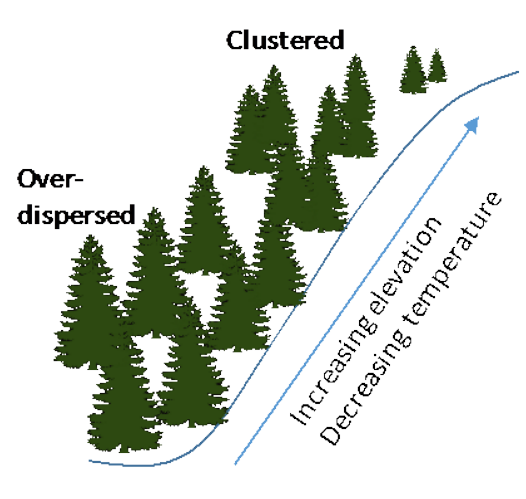

c.

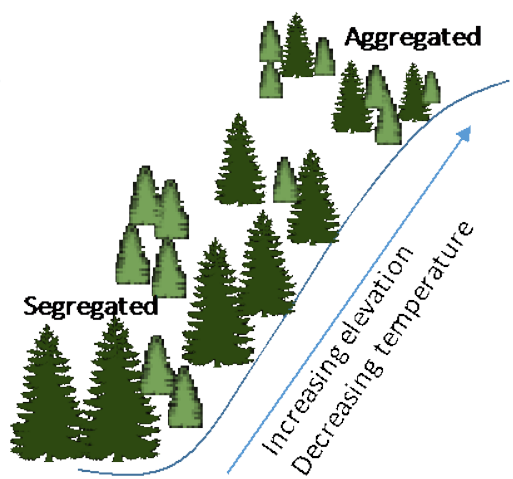

Figure 3. (a) The alpine treeline ecotone on the West Coast of New Zealand's South Island. As we move uphill, we witness a typical transition from tall, dense forest to increasingly sparse, shorter trees and shrubs, and eventually to grassland. Toward the treeline (b) growing conditions become increasingly stressful and the few remaining trees are shorter and clustered in more 
favorable microhabitats. Because environmental conditions change with increasing elevation, spatial relationships between multiple variables are likely to be anisotropic. For example, competition in the more favorable growing conditions at lower elevations results in spatial separation between adult trees (dark green) and juvenile trees (light green), whereas in the more stressful growing conditions at higher elevations, all the trees, regardless of their size, are clustered in "safe sites" where seedlings survive only where they are protected by mature trees (c).

Codispersion analysis (Rukhin and Vallejos 2008; Buckley et al. 2016) is a useful method to measure and visualize the covariation in spatial patterns between two variables, such as the position or sizes of adult and juvenile trees growing across an environmental gradient. Codispersion, which ranges from -1 to +1 , not only describes the correlation between two variables at different lags, but also accounts for the directions between them. As a result, codispersion can be used to evaluate anisotropy in the bivariate pattern, which is especially useful for analysing ecological gradients where we expect that species interactions or speciesenvironment relationships may change in their strength or other characteristics. For instance, the spatial pattern of adult trees and smaller seedlings and saplings may shift with elevation from a segregated, clustered pattern below treeline to an aggregated, clustered pattern near or above the treeline (Figure 3c).

To illustrate how codispersion analysis can be used to detect and visualize anisotropic covariation between two ecological variables, we first simulated four distinct bivariate spatial patterns that ecologists commonly encounter in the field, such as the abundances or sizes of two species along a gradient (Figure 4). The codispersion of each pair of two patterns at different spatial lags and directions are plotted as codispersion graphs (far right panels in Figure 4). As in a variogram, codispersion values are plotted for a range of spatial lags, but now these values are plotted in multiple directions. When we observe a change in the strength of the codispersion (illustrated with different colors) from one side of the graph to another, we interpret it as evidence for the presence of an anisotropic relationship between the two patterns.

In the first two simulation examples (Figures 4a and 4b), at least one of the spatial patterns is random. Thus, there is little-to-no covariation between the two patterns and the codispersion between them is very low. In contrast, if both of the simulated patterns are 
distributed as gradients in the same direction, there is positive and strong covariation at all lags and directions (Figure 4c). However, the alignment of the two patterns in the same direction means that the covariation between the variables is not anisotropic, and therefore we see no substantial directional change in codispersion across the graph. Finally, if the two patterns vary in different directions, the codispersion graph captures the expected anisotropy (Figure 4d). There is a clear change from strong negative codispersion (blue) at all lags in the positive horizontal (X) and vertical (Y) directions to a strong positive codispersion (red) in the negative horizontal and positive vertical directions. We interpret this latter result as a positive relationship at lags from about 0 to $50 \mathrm{~m}$ between the two patterns looking "northwest" from the bottom center of the plot.
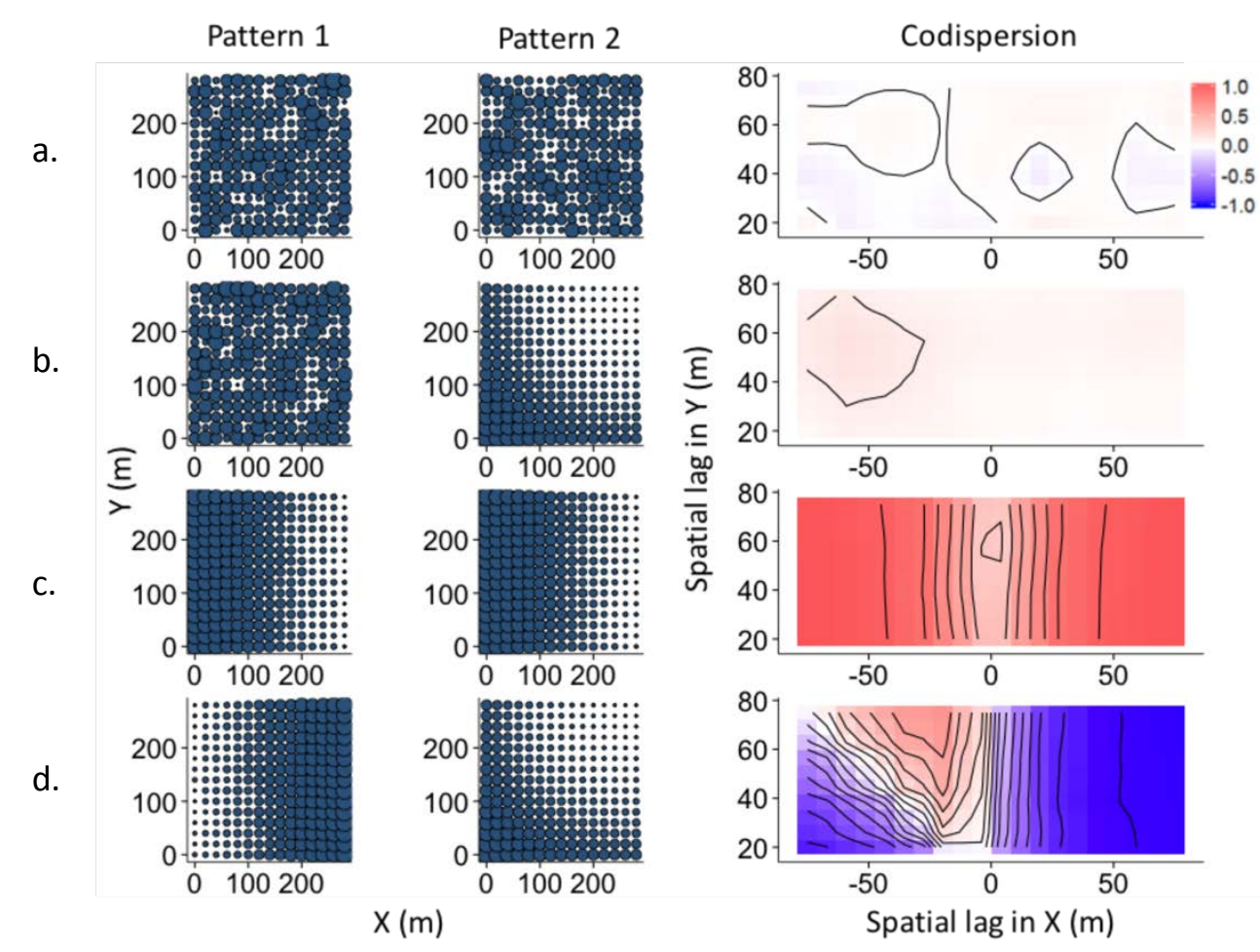

Figure 4. Simulations of bivariate spatial patterns and their resultant codispersion graphs: (a) two completely spatially random (CSR) patterns; (b) a CSR pattern and a directional pattern; (c) two directional patterns changing in the same direction; (d) two patterns changing in different directions. 
Simulations can tell us how we expect codispersion to change for contrived, albeit familiar, patterns, and so can help guide our interpretation of codispersion plots of real data. To illustrate this, we examined codispersion at treeline of diameters of mature and juvenile mountain pine trees (Pinus uncinata) in a 3,000 $\mathrm{m}^{2}$ plot located in the Spanish Pyrenees (Figure $5 a, b$ ), and of tree age and height of larch trees (Larix potaninii var. macrocarpa) in a 8,100 $\mathrm{m}^{2}$ plot located in the southeastern Tibetan Plateau (Figure 5c,d). An interesting feature of both of these treeline plots is that there has been an increase in establishment of young trees at treeline as a result of recent increases in temperature associated with climatic change (Camarero \& Gutiérrez, 2004; Liang et al., 2011). We therefore expect to detect anisotropic patterns of trees and their attributes, and the relationships between them, as we move from lower elevations to the edge of the treeline.

a.

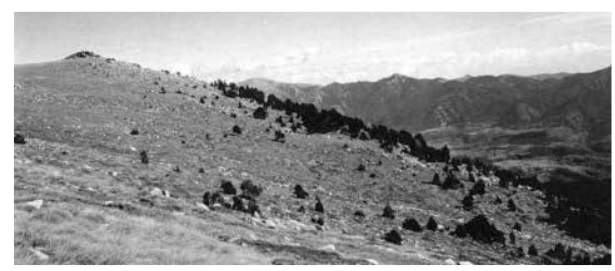

c.

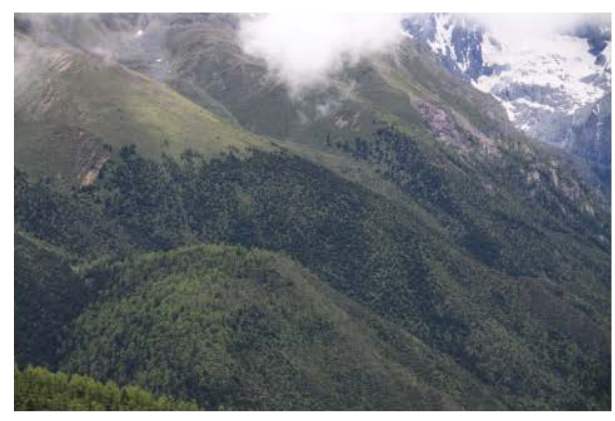

b.
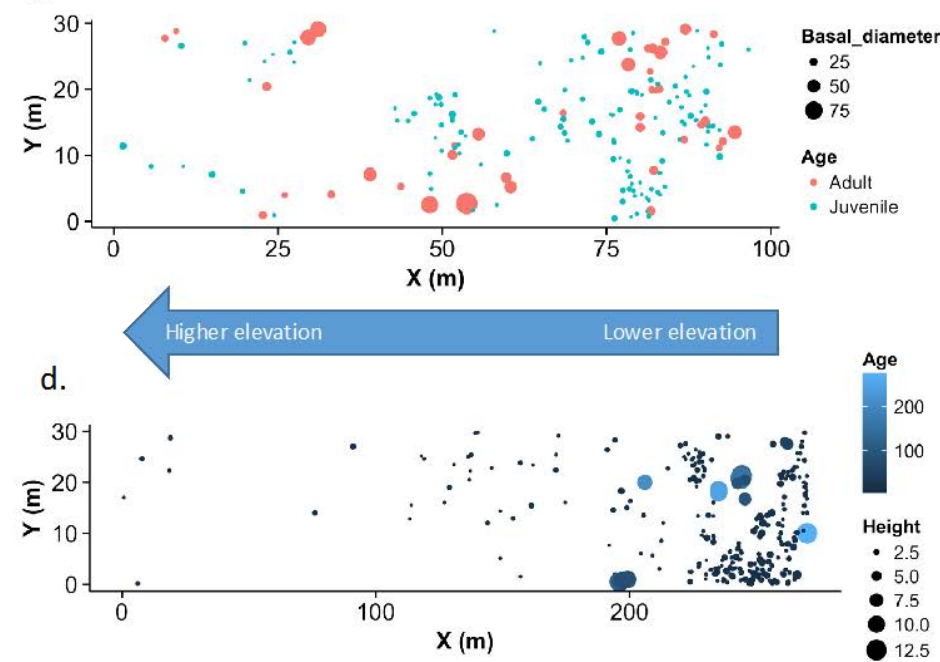

Figure 5: Photographs and maps showing tree spatial patterns of (a,b) mountain pine trees (Pinus uncinata) in a $30 \times 100-\mathrm{m}$ treeline plot in the Spanish Pyrenees and (c, d) larch trees (Larix potaninii var. macrocarpa) in a $30 \times 270$-m treeline plot in the southeastern Tibetan Plateau. The symbols in the map of the pine trees denote growth stages (adult versus juvenile) and tree diameters (sizes of symbol), whereas the map of the larch trees shows tree ages (colors) and heights (sizes of symbols). Note that both the photographs and the maps are oriented with the slope gradient along the $x$-axis, with the downhill forest on the right of the map and the uphill treeline at the left. In both cases, treeline is at the edge of the graph (at $0 \mathrm{~m}$ on the $x$-axis). 
When we look at the codispersion plots, we can observe an anisotropic relationship between the sizes of adult and juvenile trees at the Pyrenees plot (Figure 6a). There, codispersion values are low-to-negative at all spatial lags and directions in the right half of the graph (blue to white), but become increasingly positive (red) towards the top-left side of the graph (toward the treeline) at lag distances of about 20 meters. The negative codispersion in adult versus juvenile mean tree size at many lags, and particularly in the downhill direction (toward the forest on the right), probably reflects competitive processes, where the sizes of young trees are reduced when they are near to mature trees. As we move left toward the treeline, however, the younger trees are recruiting into gaps away from adults and are experiencing good growth conditions. This leads to a positive codispersion in sizes of adult and juvenile trees.

a.

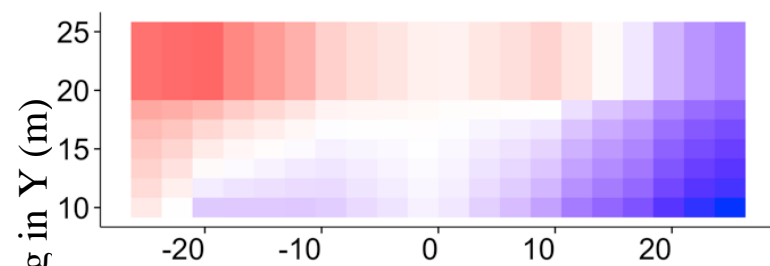

跳 $\mathrm{C}$.

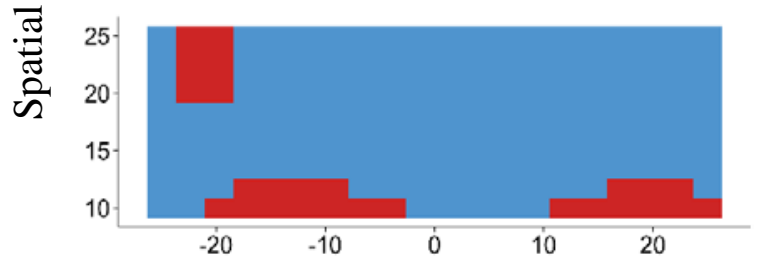

b.

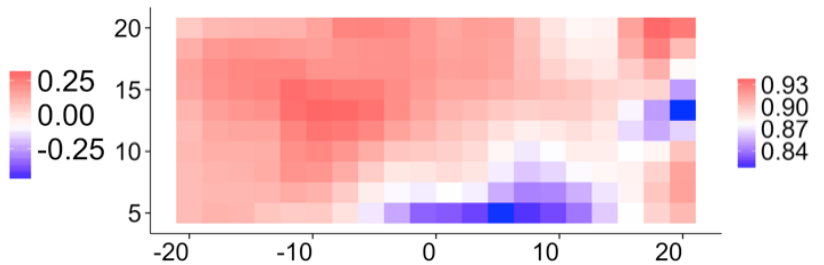

d.

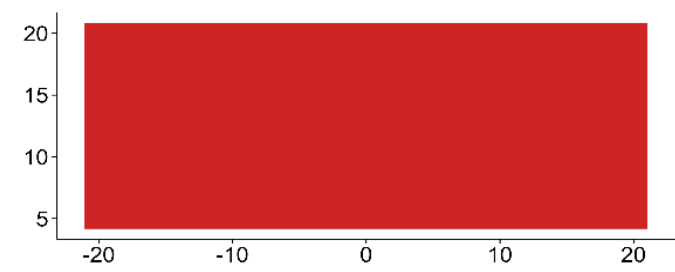

Spatial lag in X (m)

Figure 6. Codispersion graphs showing covariation between: (a) mean tree basal diameters of adult and juvenile mountain pine trees in the Spanish Pyrenees and (b) heights and ages of larch trees on the Tibetan Plateau. Graphs (c) and (d) show whether the codisperson coefficient at each lag and direction is statistically significant (red; $P<0.05$ ) or not (blue; $P>0.05$ ) in the Pyrenees and Tibetan plots, respectively. Significance is determined by comparing each observed codispersion value to that from 199 realizations of a random labeling null model, in which the attributes of one of the patterns in each plot were randomly assigned to trees for each iteration of the simulation.

The relationship between the heights and ages of larch trees on the Tibetan Plateau also is anisotropic. In its codispersion graph (Figure 6b), the covariance between tree age and height is 
positive and strong across the entire plot (the codispersion coefficient ranges from $\approx 0.8$ to 0.93 ), and as for pines in the Pyrenees, strengthens towards treeline. We interpret this result as reflecting better, open-growth conditions near treeline that result in trees whose growth in height has not been stunted by competition.

Codispersion graphs illustrate relationships between two observed patterns and so we need a way to assess how expected or unexpected these observed relationships are. One way we can do this is to use null models (Gotelli, this issue). For example, one null modeling procedure keeps one of the two observed patterns constant while randomizing either the locations or attributes of the other pattern. Following each randomization, we recalculate the codispersion. If we repeat this randomization and recalculation procedure many times, we obtain a distribution of codispersion values for each lag and direction. We then compare each observed codispersion value to the distribution of null model-derived codispersion values to assess its significance.

Figures 6c and 6d show the results of this null-model significance test for the codispersion graphs of the Pyrenees and Himalayan plots, respectively. In both cases, we used a “random labelling” null model, in which the attributes of one of the patterns in each plot were randomly assigned to trees. By reassigning attributes, this null model breaks any existing relationship between tree attributes, such as basal diameters, heights, or ages, while maintaining the underlying spatial pattern of tree locations. The results show that codispersion between diameters of adult and juvenile pine trees in the Pyrenees is statistically significant only at a few lags and directions, including at the treeline. For the majority of lags and directions, however, codispersion between adult and juvenile tree sizes is not different from what would be expected if the diameters of juvenile trees were assigned randomly to the given set of juvenile tree positions. This result might be explained by the fact that juvenile trees at this plot are quite similarly-sized, and thus randomizing their sizes relative to adult trees does not generate much significant variation in the adult-juvenile codispersion relationship. In contrast, for larch trees in the Tibetan Plateau, the codispersion of heights and ages is significantly different from random expectation in all lags and directions, reflecting the strong, intrinsic relationship between tree age and height. In this example, the random labelling null model has the effect of assigning a range of height values to both old and young trees, thus truly randomizing the relationship between height and age, causing highly significant departures from the strong observed codispersion 
between these two variables. These examples highlight how the choice of null model determines the types of interpretations that can be made using the codispersion analysis, and that multiple null models often need to be explored.

In conclusion, codispersion analysis allows us to detect and describe relatively subtle changes in bivariate relationships across environmental gradients, which is something that has traditionally been hard to tackle with spatial pattern analysis. When combined with null models, we can test how unexpected these observed patterns are. Our ongoing research is exploring how codispersion applies to a wide range of spatial patterns and null models, and how to use this information to improve models for spatial analysis and forecasting.

\section{References cited}

Bivand RS., Pebesma EJ., Gomez-Rubio V. 2008. Applied spatial data analysis with R. Springer, New York.

Buckley, HL., Case, BS., Ellison, AM. 2016. Using codispersion analysis to characterize spatial patterns in species co-occurrences. Ecology. In press.

Camarero JJ \& Gutiérrez E. 2004. Pace and pattern of recent treeline dynamics: response of ecotones to climatic variability in the Spanish Pyrenees. Climatic Change 63: 181-200.

Liang, E, Wang Y, Eckstein D, and Luo T. 2011. Little change in the fir tree-line position on the southeastern Tibetan Plateau after 200 years of warming. New Phytologist 190:760-769.

Rukhin, AL. and Vallejos, R. 2008. Codispersion coefficients for spatial and temporal series. Statistics and Probability Letters 78: 1290-1300. 